\title{
Generalized D-Forms Have No Spurious Creases
}

\author{
Erik D. Demaine • Gregory N. Price
}

Received: 21 December 2007 / Revised: 28 July 2009 / Accepted: 28 July 2009 /

Published online: 16 September 2009

(C) Springer Science+Business Media, LLC 2009

\begin{abstract}
A convex surface that is flat everywhere but on finitely many smooth curves (or seams) and points is a seam form. We show that the only creases through the flat components of a seam form are either between vertices or tangent to the seams. As corollaries we resolve open problems about certain special seam forms: the flat components of a D-form have no creases at all, and the flat component of a pita-form has at most one crease, between the seam's endpoints.
\end{abstract}

Keywords Convex bodies · Alexandrov's theorem · Paper folding · Gluing

\section{Introduction}

Given any metric space $S$ with the topology and local geometry required of the surface of a convex three-dimensional body, there is exactly one convex body up to isometry whose surface has the intrinsic geometry of $S$. This is the endpoint of a line of research pursued in the middle of the last century by Alexandrov and Pogorelov [1], and it implies a strong correspondence between the geometry of a convex body and the intrinsic geometry of its surface. On the other hand, the exact nature of this correspondence is not yet well understood-which properties in the surface geometry imply what properties in the body geometry, and vice versa.

E.D. Demaine partially supported by NSF CAREER award CCF-0347776, DOE grant DE-FG02-04ER25647, and AFOSR grant FA9550-07-1-0538.

G.N. Price partially supported by an NSF Graduate Fellowship.

E.D. Demaine · G.N. Price $(\bowtie)$

MIT Computer Science and Artificial Intelligence Laboratory, 32 Vassar St., Cambridge, MA 02139, USA

e-mail: price@mit.edu

E.D. Demaine

e-mail: edemaine@mit.edu 
For example, if $S$ is a $D$-form, obtained by sewing together two smooth convex shapes of the same perimeter, then even for this special case the most basic questions are open. These forms were invented by an artist $[8,9]$ and introduced into the literature in [6]. The latter study poses three problems: (1) when is the D-form the convex hull of a space curve, (2) when are the two pieces free of creases, and (3) how can one compute the D-form numerically from the two shapes. A later treatment [3] suggests an informal argument for Problem 1 (arguing that the D-form is always the convex hull of its seam) and leaves Problems 2 and 3 open. The same book [3] introduces also a related special case where $S$ is obtained by sewing up a single smooth convex shape along its boundary in one seam, calling these pita-forms and suggesting, based on paper experiments, that pita-forms might never have creases.

We resolve Problems 1 and 2: both D-forms and pita-forms are always the convex hull of their seams, and (excluding the seam) D-forms are always free of creases but a pita-form may have one crease. Our results apply to a natural generalization of both D-forms and pita-forms, the seam form, which roughly consists of intrinsically flat pieces joined along finitely many seams. Because the original sources of the problem are stated informally, we first introduce precise definitions of D-form, crease, etc., that we believe capture the intuitive picture. Then we show the following theorems:

Theorem 1 Every three-dimensional convex body is the convex hull of the nonflat points on its surface.

Corollary 2 Every seam form is the convex hull of its seams and vertices.

Theorem 3 In a flat component of a seam form, every crease lies on a line segment composed of creases, and each endpoint of such a segment is either a strict vertex or a point of tangency to a seam.

Corollary 4 The flat components of a D-form are without creases; in the flat component of a pita-form, the only crease(s), if any, make up the line segment between the endpoints of the seam.

Intuitively the line segment between a pita-form's endpoints in Corollary 4 should be thought of as one "crease"; it is a consequence of our definitions, below, that this segment may be arbitrarily subdivided into several segments we call creases.

Problem 3, to efficiently compute the three-dimensional shape of a D-form or seam form from its two-dimensional intrinsic geometry, has now been largely resolved. To make this problem well posed, one needs a finite representation of the input geometry, which is most naturally done by a piecewise-linear or polyhedral approximation. With considerable effort, the problem of reconstructing a three-dimensional convex polyhedron from its intrinsic geometry can be reduced to the solution of a highdimensional ordinary differential equation [2]. The numerical solution of this equation appears to be achievable efficiently in practice, and is provably achievable within pseudopolynomial time [5].

We introduce terminology in Sect. 2, prove Theorem 1 and its Corollary 2 in Sect. 3, and prove Theorem 3 and its Corollary 4 in Sect. 4. In Sect. 5, we describe counterexamples that show the necessity of some of the hypotheses in our results. 
Fig. 1 A D-form, constructed by sewing together two ellipses (at left). The solid line is the seam, and the dotted lines are the false edges from projection into the page

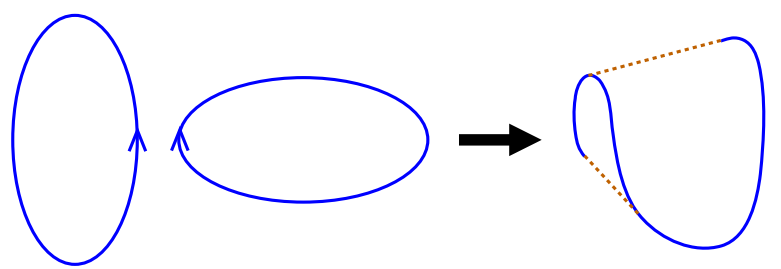

\section{Background and Notation}

For us a surface is a metric 2-manifold embedded in $\mathbb{R}^{3}$. The surface is $C^{k}$ if the manifold and its embedding are $C^{k}$. The surface is piecewise- $C^{k}$ if it can be decomposed as a complex of vertices, $C^{k}$ open edges, and $C^{k}$ open regions.

A good surface is a piecewise- $C^{2}$ surface. A good surface $S$ therefore decomposes into a union of $C^{2}$ surfaces $S_{i}$, called pieces, $C^{2}$ edges $\gamma_{j}$, which we call semicreases, and vertices. If $S$ is itself $C^{1}$ everywhere on a semicrease, we call it a proper semicrease; otherwise it is a crease. (This conservative definition of crease, where some parts may be $C^{1}$ but not $C^{2}$, only broadens our characterization of creases in seam forms.) Note that this definition is not intrinsic, because it relies on the decomposition of $S$ into $C^{2}$ pieces; by describing a surface by a different decomposition it is possible to add proper semicreases or to subdivide creases. This reliance on a piecewise $C^{2}$ decomposition avoids irrelevant issues from real analysis.

A point on a surface is flat if it has a neighborhood isometric to a region in the plane. A surface or part of a surface is called flat if all of its points are flat.

A surface $S$ is convex if $S \subseteq \partial X$ for some bounded convex body $X$ in $\mathbb{R}^{3}$. A normal to a convex body $X$ at a point $x$ is a unit vector $n$ with $n \cdot x=\sup _{x^{\prime} \in X} n \cdot x^{\prime}$. The relation between points on the boundary of $X$ and their normals is traditionally called the Gauss map, though it need not be a map-one point may have many normals. We write $G(x)$ for the normals at $x$, and $G(U)$ for all the normals to any point in $U \subset X$. Observe that $G(x)$ is always a convex subset of the sphere.

A consequence of Gauss' celebrated Theorema Egregium [4] is that a convex surface $U$ is flat just if $G(U)$ has zero area. If $G(x)$ has positive spherical area, then we call $x$ a strict vertex. The $C^{2}$ condition prevents a strict vertex $x$ from being on a semicrease or piece, so for good surfaces, strict vertices are indeed vertices.

A seam form $S$ is a good convex surface in which each piece $S_{i}$ is flat. It is simple to verify that such a surface decomposes uniquely into maximal connected flat open subcomplexes, which we call flat components, and some leftover semicreases and vertices, the connected components of which we call seams.

A convex seam form is a seam form in which each flat component is isometric to a convex plane region, and a convex smooth seam form is a convex seam form in which these regions have smooth $\left(C^{\infty}\right)$ boundary. A convex smooth seam form with one flat component is called a pita form, and with two flat components is called a D-form. See Figs. 1 and 3.

Given a convex body $X$ and a point $x \in X$, we say that a line $\ell$ traverses $x$ if $x \in \ell$ and some open neighborhood of $x$ on $\ell$ is contained in $X$. An extreme point of $X$ is a point $x \in X$ not traversed by any line. 


\section{Convex Hull}

In this section, we prove Theorem 1, that every three-dimensional convex body is the convex hull of the nonflat points on its surface.

From convex geometry we have the following characterization of the minimal set from which a convex body can be recovered as the convex hull: we need only the extreme points.

Theorem 5 (Minkowski's Theorem) Every convex body in $\mathbb{R}^{n}$ is the convex hull of its extreme points.

The proof is a straightforward induction on the dimension of the body and can be found as Corollary 1.4.5 in Schneider's textbook [7].

It remains to describe the extreme points of a seam form. To do so, we begin with the following proposition:

Proposition 6 If $p$ is an extreme point of the convex body $X$, then for every open neighborhood $U$ of $p$ in $\mathbb{R}^{n}$, some hyperplane has $p$ strictly on one side and all of $X \backslash U$ strictly on the other.

Proof This is Lemma 1.4.6 in [7]. For completeness we give the proof.

Because $p$ is an extreme point, it cannot by definition be the convex combination of any two other points in $X$. Therefore, it is not the convex combination $a_{1} x_{1}+\cdots+$ $a_{k} x_{k}$, with all $a_{i}>0$, of any $k$ other points in $X$, because otherwise we would have $p=\left(1-a_{k}\right)\left(\frac{a_{1}}{1-a_{k}} x_{1}+\cdots+\frac{a_{k-1}}{1-a_{k}} x_{k-1}\right)+a_{k} x_{k}$, and certainly the convex combination $\frac{a_{1}}{1-a_{k}} x_{1}+\cdots+\frac{a_{k-1}}{1-a_{k}} x_{k-1}$ is a point in $X$. In other words, $p$ lies outside the convex hull of $X \backslash\{p\}$, and consequently outside the convex hull $Y$ of $X \backslash U$.

Now $Y$ is itself a convex body, and $p$ a point outside it. By the Separating Hyperplane Theorem, some hyperplane strictly separates them, and because $X \backslash U \subset Y$, it strictly separates $p$ and $X \backslash U$ as required.

Proposition 7 On the surface of a convex body, there are no flat extreme points.

Proof Suppose some extreme point $p$ of a convex body $X$ was flat, with a neighborhood $S \subset \partial X$ isometric to a plane region. Let $U$ be an open neighborhood of $p$ in $X$ with $U \cap \partial X \subset S$. Let the hyperplane $H$ guaranteed by Proposition 6 separate $X$ into convex bodies $C$ and $Y$ with $p \in C$, and let $D=C \cap \partial X$. Because $C \subset U$, we have $D \subset S$ so that $D$ is flat.

Now consider the normals to $X$ along the portion $D$ of its surface. Let $d$ be the distance from $p$ to $H$, and let $r$ be the maximum distance from the projection of $p$ onto $H$ to any point in $H \cap X$. Then any plane through $p$ and making an angle at most $\theta=\tan ^{-1}(d / r)$ to $H$ fails to intersect $H \cap X$ and therefore fails to intersect $Y$. Therefore, the normals to these planes, covering a spherical area of $2 \pi(1-\cos \theta)>0$, all are normals to $X$ somewhere on $D$. This gives $G(D)$ a positive area, contradicting that $D \subset S$ is flat. 
Theorem 1 is now immediate from Theorem 5 and Proposition 7, and Corollary 2 follows.

\section{Creases}

In this section, we prove Theorem 3, characterizing the possible creases of a seam form.

Proposition 8 Let $\gamma$ be a crease in a flat component of a seam form $S$. Then $\gamma$ lies on a line segment $[p, q]$ between endpoints $p$ and $q$ that lie on seams, and the whole segment is composed of creases and vertices.

Proof Let $S_{1}$ and $S_{2}$ be the open pieces bordered by $\gamma$ in the decomposition of the good surface $S$, and let $x \in \gamma$ be a point at which $S$ is not $C^{1}$. Then $S_{1}$ and $S_{2}$ are $C^{2}$ surfaces, so they have normals $n_{1}$ and $n_{2}$ at $x$, and because $S$ is not $C^{1}$ at $x$, these normals are distinct. Therefore, $G(x)$ contains at least two distinct vectors.

By Proposition 7, $x$ must be traversed by some line $\ell$, so that $\ell \cap S=[p, q]$ for some $p$ and $q$. Necessarily $\ell$ is perpendicular to all of $G(x)$, so for each $y \in[p, q]$ and each normal $n \in G(x), n \cdot y=n \cdot x=\sup _{x^{\prime} \in X} n \cdot x^{\prime}$ and $n$ is a normal of $y$. Therefore, each $G(y)$ contains $G(x)$, and so like $G(x)$ has at least two distinct vectors.

The multiple normals in $G(x)$ and hence in each $G(y)$ determine a unique perpendicular line, so that no other line may traverse any point of $[p, q]$. In particular, no line traverses $p$ or $q$, so by Proposition 7, these points are not flat and must lie on seams or vertices.

At the same time, because a $C^{1}$ surface has only one normal at each point, no point of $[p, q]$ can be on a $C^{2}$ piece or a semicrease. The whole segment is therefore made up of creases and (nonstrict) vertices. Because a crease is defined from a cellcomplex decomposition, only one crease runs through a given point, so because $\gamma$ runs through $x$ it must be one of the creases making up $[p, q]$.

In order to analyze the Gauss map at seam and vertex points, we introduce some additional notation. Let $x \in S$ be incident to a 1- or 2-cell $C$, a (semi)crease or piece. Then we define

$$
G_{C}(x)=\bigcap_{x \in U \text { open }} \operatorname{closure}(G(C \cap U))
$$

as the Gauss map at $x$ on $C$. For comparison, observe that

$$
G(x)=\bigcap_{x \in U \text { open }} \operatorname{closure}(G(U))
$$

because the relation $G$ is closed, and in particular $G_{C}(x) \subseteq G(x)$.

Proof of Theorem 3 Let $\gamma$ be a crease in a flat component of a seam form $S$. By Proposition $8, \gamma$ lies on a segment $[p, q]$ composed of creases and vertices and whose 
endpoints lie on seams. It remains to prove that if an endpoint, say $p$, lies on a seam and is not a strict vertex, then the seam is tangent to $[p, q]$.

Let $G_{\gamma}(p)$ be the great circular arc $m n$, and let the pieces of $S$ bordering $\gamma$ be $S_{1}$ and $S_{2}$. By continuity, $G_{S_{1}}(p) \ni m$ and $G_{S_{2}}(p) \ni n$ (possibly after exchanging the names $m, n)$, and because $m \neq n$ the Gauss map at $p$ on at least one of the cells $C$ surrounding $p$ from $S_{1}$ to $S_{2}$ apart from $\gamma$ must be a positive-length spherical curve in order to complete the path from $m$ to $n$. If $p$ is not a strict vertex, then $G(p)$ is a convex spherical shape of zero area, so it is a great circular arc, and $G_{C}(p) \subseteq G(p)$ is also a great circular arc. If $C$ is a piece, then $G_{C}(p)$ is either a singleton or a curve not lying on a great circle, because a great-circle Gauss map makes parallel rule lines that cannot converge at $p$. Therefore, $C$ is a semicrease. Because $G(C)$ must be more than a single point, $C$ is a crease, and to make the Gauss map lie within the arc $G(p)$, $C$ must be tangent to $[p, q]$ as required. Finally, because $p$ is the endpoint of the intersection of the line $p q$ with $S$, the crease $C$ must not be a line segment, so by Proposition 8 , it is actually part of the seam and the proof is complete.

Of course, in a convex plane region, no line segment in the interior is tangent to the boundary, from which follows a corollary about convex seam forms.

Corollary 9 In a convex seam form, every crease in a flat component is on a line segment between two strict vertices.

Finally, in a convex smooth seam form such as a pita-form or a D-form, the requirement of smoothness sharply limits the possible configurations. By (local) convexity, no vertex can be incident to three or more semicreases as part of its seam, and a vertex through which a seam passes cannot be a strict vertex. Consequently, a pita-form must have a single path for its seam and just two strict vertices located at the seam's endpoints, and a D-form must have a single cycle for its seam and no strict vertices. Corollary 4 follows.

\section{Counterexamples}

We have required the flat components of a D-form to be convex. We could relax this requirement, requiring instead only that the metric space resulting from joining the two components be locally convex, and the Alexandrov-Pogorelov theorem would still guarantee a unique convex embedding in three-dimensional space. Of course, Corollary 2 would still guarantee that the resulting body would be the convex hull of its seam, but it turns out that Corollary 4, whose conditions would no longer be satisfied, really would fail in its conclusion: one can construct a "D-form" under this relaxed definition which contains creases in its flat components. Indeed, it is not hard to construct such an example, if one keeps in mind Theorem 3 that the offending crease must be tangent to a seam; see Fig. 2. It would be interesting to determine under exactly what conditions a seam form whose flat components do not correspond to convex plane regions has a crease. 
Fig. 2 A "D-form" with a relaxed convexity condition. The solid dark line is the seam, the dotted lines are false edges from projection onto the page, and the solid light lines are the creases through a flat component. Actual D-forms have no such creases
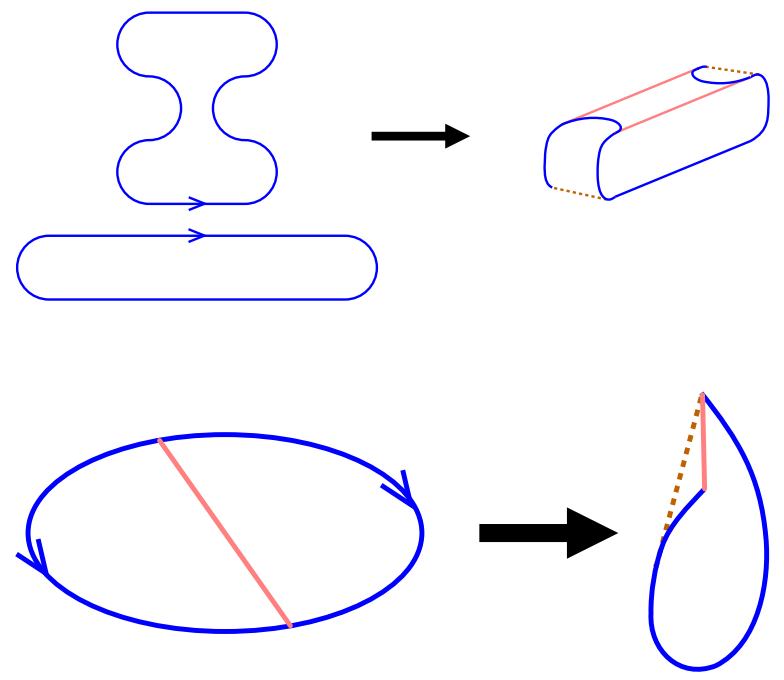

Fig. 3 A typical pita-form. A crease runs between the endpoints of the seam

For pita-forms, we have concluded in Corollary 4 that a pita-form may have at most one crease. Indeed this is tight, and it is easy to construct an example pitaform with a crease; see Fig. 3. This possibility of creases therefore represents a real difference from D-forms. It represents also a contrast from the appearance of the natural paper experiments, which led the authors first introducing pita-forms ([3]) to suggest that pita-forms might never have creases. In fact, by applying Corollary 2 , it is not difficult to see that the pita-form depicted in Fig. 23.14 of [3] must have a crease. In our reproduction, the physical paper indeed does not crease, and the seam has gaps at the endpoints that in retrospect explain the divergence between this experiment's behavior and the mathematical pita-form.

On the other hand, we do not know of a construction for a pita-form without a crease. It appears to be an open problem to find such a construction, or to prove that in every pita-form the line segment between the endpoints is, in fact, a crease.

For some theorems of the same flavor as our results here, one might hope to obtain proofs by showing that the desired properties hold of convex polyhedra, which are relatively concrete and amenable to reasoning, and then that they carry over to general convex bodies as limits of polyhedra. Indeed, this is the approach taken in [3] to argue for Corollary 2 for the case of D-forms. Unfortunately, this approach does not hold as widely as one might like. In particular, Corollary 9 cannot be proven by a limiting argument of the obvious form, even when restricted to D-forms. When each flat component of the D-form is approximated by a sequence of polygons, it is possible for the dihedral angles inside the components to approach positive limits, even when the angles of the polygonal approximations are required to converge to zero. For example, in the D-form obtained from two circular disks (which is just the double cover of a disk), the components may be approximated by regular $n$-gons for increasing $n$, and the resulting approximations to the D-form may be antiprisms of two smaller $n$-gons and $2 n$ triangles. In this approximation sequence, the dihedral angles between each $n$-gon and its $n$ neighboring triangles approach $\pi / 3$, not zero, 
even though they lie inside the flat components. For this and other reasons, we have chosen direct proofs that attack the general case of convex bodies.

Acknowledgements We thank Jonathan Kelner, Joseph O'Rourke, and Johannes Wallner for helpful discussions, and the anonymous referees for helpful comments.

\section{References}

1. Alexandrov, A.D.: Convex Polyhedra. Springer, Berlin (2005). See especially note 21, p. 189

2. Bobenko, A.I., Izmestiev, I.: Alexandrov's theorem, weighted Delaunay triangulations, and mixed volumes. Ann. Inst. Fourier 58(2), 447-505 (2008). arXiv:math.DG/0609447

3. Demaine, E.D., O'Rourke, J.: Geometric Folding Algorithms. Cambridge University Press, Cambridge (2007). pp. 352-354

4. Gauss, C.F.: General Investigations of Curved Surfaces (1827). Morehead and Hiltebeitel, tr. Princeton (1902)

5. Kane, D., Price, G.N., Demaine, E.D.: A pseudopolynomial algorithm for Alexandrov's theorem. In: Algorithms and Data Structures Symposium (WADS) (2009). arXiv:0812.5030

6. Pottmann, H., Wallner, J.: Computational Line Geometry. Springer, Berlin (2001). p. 418

7. Schneider, R.: Convex Bodies: The Brunn-Minkowski Theory. Cambridge University Press, Cambridge (1993)

8. Sharp, J.: D-Forms: Surprising New 3-D Forms from Flat Curved Shapes. Tarquin Publications, St. Albans (2009)

9. Wills, T.: DForms: 3D forms from two 2D sheets. In: Sarhangi, R., Sharp, J. (eds.) Bridges: Mathematical Connections in Art, Music, and Science, pp. 503-510. London 